\title{
Are children born from singleton pregnancies conceived by ICSI at increased risk for congenital malformations when compared to children conceived naturally? A systematic review and meta-analysis
}

\author{
Celeste Lacamara ${ }^{1}$, Carolina Ortega², Sonia Villa², Ricardo Pommer ${ }^{2}$, Juan Enrique Schwarze ${ }^{2}$ \\ ${ }^{1}$ University of Santiago, Chile \\ ${ }^{2}$ Reproductive Medicine Unit at Clinica Monteblanco, Santiago, Chile
}

\begin{abstract}
Since 1992, the development of intra-cytoplasmic sperm injection (ICSI) has allowed infertile couples and couples affected by severe male factor infertility in particular, many of which with a history of failed traditional IVF, to become parents. This has generated considerable controversy over the safety of the procedure for the offspring. This systematic review seeks to determine whether evidence indicates that the use of ICSI increases the risk of congenital malformation in children born from singleton pregnancies versus naturally conceived children. Twenty-one of the 104 publications listed in the literature search were included in the analysis. Observational studies reported mostly an increased risk for congenital malformation; the risk of congenital malformations is $7.1 \%$ in ICSI and $4.0 \%$ in the general population (OR 1.99 (95\% CI $[1.87-2.11])$. However, attributing higher risk solely to ICSI might seem far-fetched, as in vitro and simulation procedures, patient diseases, and ICSI indication may also be associated with higher risk of malformation.
\end{abstract}

Keywords: ICSI, congenital malformations, pregnancy.

\section{INTRODUCTION}

Assisted reproductive technologies such as in vitro fertilization (IVF) and intra-cytoplasmic sperm injection (ICSI) yield a higher incidence of preterm neonates, low birth weight, and multiple births (Hansen et al., 2005). ICSI has been used successfully since 1992 (Massaro et al., 2015) and allowed the treatment of a much wider range of conditions that cause infertility, especially male factor infertility and the frustrating and often intractable unexplained infertility. This method has given hope to millions of people unable to achieve pregnancy through traditional IVF. However, the use of ICSI, in virtue of its invasive nature and the types of conditions it treats, has raised concerns about the increased risk of congenital malformations. Fundamentally, there are three factors associated with ICSI that may influence the risk of malformation: chromosomal abnormalities in the gametes used; drugs used in ovarian hyperstimulation; and the direct injection of sperm into the oocyte. The latter eludes natural selection on the membrane of the maternal gamete, and may damage the egg and introduce foreign materials (e.g.: culture medium) into the cell (Retzloff \& Hornstein, 2003).

Congenital malformations are innate structural or functional abnormalities. Their origin may be genetic or multifactorial, but in most cases the cause is unknown. They are categorized as major or minor according to the European Registry of Congenital Anomalies (EUROCAT). Major malformations are physical abnormalities that are incompatible with life, require surgical repair, and/or cause functional impairment (Bertelsmann et al., 2008). Various researchers have explored the association between ICSI and risk of major malformations. Tararbit et al. (2013) found that ICSI was associated with a significant increase in the risk of Tetralogy of Fallot, but not of other congenital heart diseases. Ericson \& Källén (2001) reported increases the risk of neural tube defects, atresia of the digestive tract, omphalocele, and hypospadias. Wennerholm et al. (2000) described similar findings. However, Jwa et al. (2015) have not found increased risk of congenital malformations associated with ICSI, even after subgroup analysis considering the stage of embryonic development at the time of transfer and/or the use of cryopreservation.

Given this controversy, it is difficult to advise couples of the possible implications of ICSI. Therefore, this systematic review aimed to determine whether ICSI is associated to increased risk of congenital malformations.

\section{MATERIALS AND METHODS}

A search was performed in three databases (PubMed, Google Scholar, and Trip database) using extensive criteria encompassing primary studies that included in any field the following keywords: "congenital anomalies," "birth defects," "congenital malformations," and "ICSI". The search included studies published from January of 2000 to May of 2016. The inclusion criteria were: publications in English or Spanish; singleton pregnancy; frequency of malformations in the ICSI group and in naturally conceived controls. The exclusion criteria were: use of preimplantation genetic diagnosis; secondary education; lack of a control group. The extracted data were transferred to a standard form, which included reference and number of healthy patients and patients diagnosed with malformations in the ICSI and control groups.

Statistical analysis was performed with STATA (Statacorp, USA). The heterogeneity between the studies was assessed using the Chi square test. A fixed-effect model was used to perform the meta-analysis, with the Mantel-Haensze test. Odds ratios (OR) with their corresponding $95 \%$ confidence intervals (95\% CI) were calculated for each publication analyzed, based on the raw data. For the sake of improving comprehension, the results were shown in a forest plot.

\section{RESULTS}

Each of the searched databases yielded the following number of references: PubMed with 634 titles; Google Scholar system with 8,960 titles; and Trip Database with 184 titles. Of these, 563 abstracts were reviewed and 104 were selected for further analysis. Twenty-one of them were included in our review and meta-analysis. Figure 1 shows the reasons for exclusion.

Of the 21 papers reviewed, four originated in Australia, one in Iran, three in Germany, one in Sweden, three in Belgium, one in Denmark, one in Finland, one in Israel, one in Hungary, one in the Netherlands, one in the United Kingdom, and three in multiple countries. The oldest study 


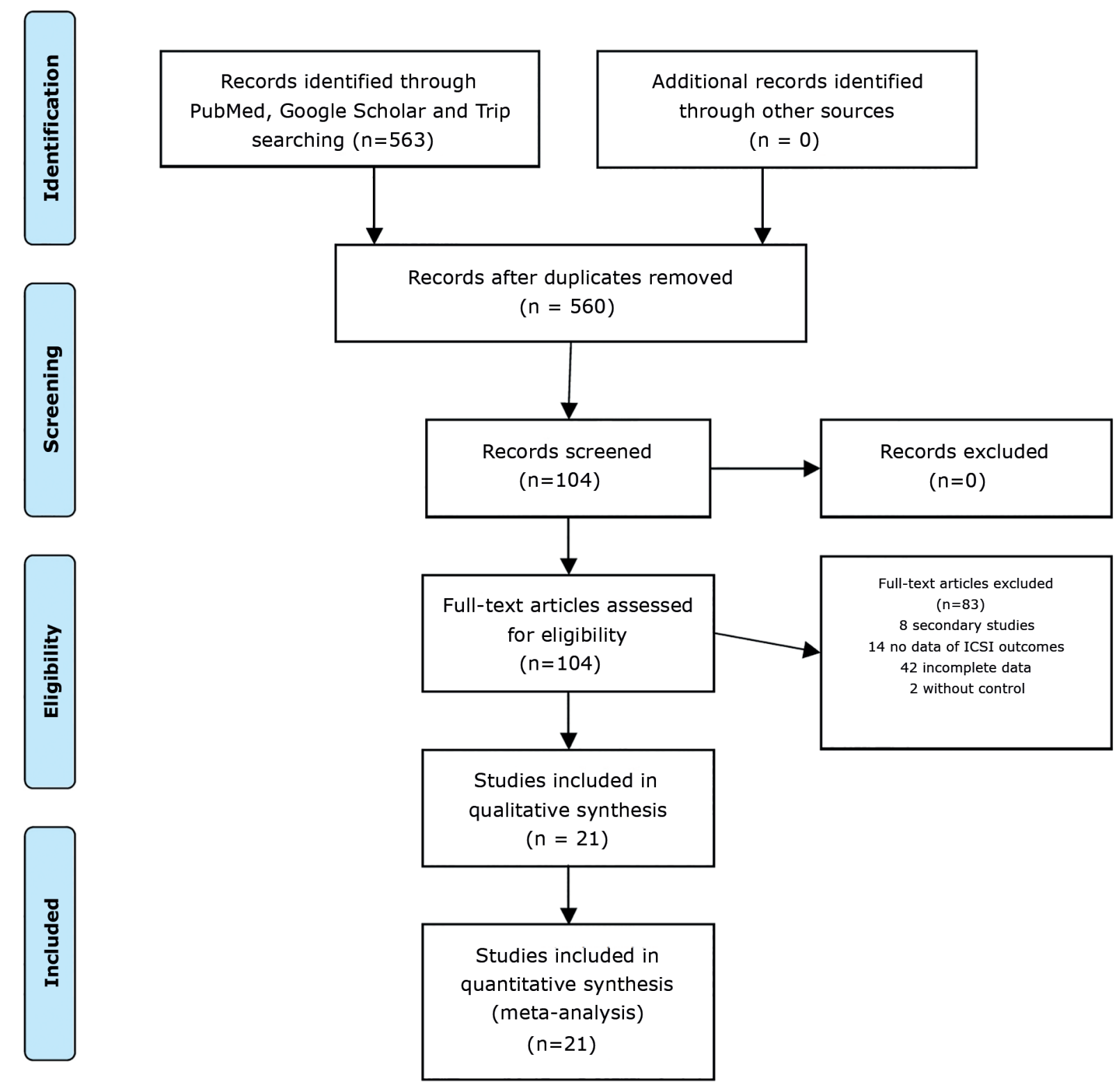

Figure 1. Flow Diagram.

was published in 2001 (Sutcliffe et al., 2001) and the most recent in October 2013 (Farhangniya et al., 2013). The number of children with malformations in the ICSI group ranged from six to 488 (total: 1,959), and in the control group from five to 80,881 (total: 117,463 ).

In most of the publications, the authors adjusted the data according to the variables that might have affected the outcomes: seventeen adjusted their data by maternal age (Hansen et al., 2002; Farhangniya et al., 2013; Bonduelle et al., 2005; Sanchez-Albisua et al., 2007; Halliday et al., 2010; Davies et al., 2012; Källén et al., 2005; Belva et al., 2007; Fedder et al., 2013; Ludwig et al., 2009; Ombele et al., 2005; Hansen et al., 2012; Klemetti et al., 2005; Farhi et al., 2013; Bonduelle et al., 2004; Katalinic et al., 2004; Knoester et al., 2008); seven for paternal age (Bonduelle et al., 2005; Davies et al., 2012; Ludwig et al., 2009; Place \& Englert, 2003; Bonduelle et al., 2004; Katalinic et al., 2004; Knoester et al., 2008); ten for parity (Hansen et al., 2002; Halliday et al., 2010; Davies et al.,
2012; Källén et al., 2005; Sutcliffe et al., 2001; Ombele et al., 2005; Hansen et al., 2012; Klemetti et al., 2005; Bonduelle et al., 2004; Knoester et al., 2008); seven for socio-economic level (Davies et al., 2012; Belva et al., 2007; Ludwig et al., 2009; Sutcliffe et al., 2001; Place \& Englert, 2003; Klemetti et al., 2005; Knoester et al., 2008); eleven for sex of the child (Hansen et al., 2002; Farhangniya et al., 2013; Halliday et al., 2010; Davies et al., 2012; Belva et al., 2007; Fedder et al., 2013; Sutcliffe et al., 2001; Hansen et al., 2012, Klemetti et al., 2005; Farhi et al., 2013; Funke et al., 2010); six for smoking during pregnancy (Belva et al., 2007; Ludwig et al., 2009; Sutcliffe et al., 2001; Hansen et al., 2012, Bonduelle et al., 2004; Knoester et al., 2008); three for use of medication during pregnancy (Farhangniya et al., 2013; Belva et al., 2007; Knoester et al., 2008); two for drinking during pregnancy (Sutcliffe et al., 2001; Bonduelle et al., 2004); one for period of infertility (Place \& Englert, 2003); and three for the presence of malformations in an elder sibling 
(Hansen et al., 2002; Källén et al., 2005; Katalinic et al., 2004).

The main outcome measured was birth defects in ten publications, while 11 papers were designed to assess general perinatal risks. Of the 21 articles reviewed, 19 reported increased risk of birth defects, but only 13 had statistically significant results (Hansen et., 2002; Bonduelle et al., 2005; Sánchez-Albisua et al., 2007; Halliday et al., 2010; Davies et al., 2012; Källén et al., 2005; Belva et al., 2007; Fedder et al., 2013; Hansen et al., 2012; Klemetti et al., 2005; Farhi et al., 2013; Funke et al., 2010; Katalinic et al., 2004). Two studies reported that ICSI was not associated with increases in congenital malformations, but none were statistically significant (Sutcliffe et al., 2003; Place \& Englert, 2003).

In a retrospective cohort study, Hansen et al. (2002) analyzed data from pregnancies of at least 20 weeks of ongoing gestation and pregnancy losses due to fetal abnormalities resulting from ICSI or IVF between 1993 and 1997. The ICSI group had 18 (9.7\%) malformed fetuses out of a total of 186 singleton pregnancies; the control group had $164(4.2 \%)$ cases of malformation from a total of 3,906 naturally conceived infants. OR 3.63 (95\% CI= [1.78-7.41]).

Farhangniya et al. (2013) performed a historical cohort study from January 2008 to December 2010, in which neonates were evaluated according to the subtype of Assisted Reproductive Technology (ART) used and compared to children born from pregnancies without ART. The data reported on singleton births showed that $17(6.5 \%)$ of 263 ICSI infants had malformations. In the group of children born without ART, $29(4.4 \%)$ of 652 were reported to have malformations. OR 1.52 (95\% CI [0.792.93]).

Bonduelle et al. (2005) published a controlled retrospective cohort study involving five European countries, in which 5405 -year-old children conceived by ICSI were compared to 538 naturally conceived children of the same age. In the ICSI group, 63 (4.2\%) children had major congenital malformations. The comparison against controls revealed an OR of 1.76 (95\% CI [1.34-2.29]). The high rate of malformations observed was partially due to an excess of urogenital malformations in males. When minor malformations were added to the analysis, the number of affected ICSI and naturally conceived children increased to $178(33 \%)$ and $117(22 \%)$, respectively.

Sanchez-Albisua et al. (2007) published a clinical followup study of children born after ICSI and controls born after 35 weeks of gestation from singleton pregnancies between August 1996 and November 2001. Major and minor malformations were found in $16 / 34$ (47\%) of the ICSI children and in $8 / 39(20 \%)$ of the naturally conceived subjects. OR 3.27 (95\% CI [1.23-8.66]).

Halliday et al. (2010) published a retrospective cohort study on birth defects in which 3,634 singleton pregnancies after ICSI occurred between 1991 and 2004 in Victoria, Australia, were compared to 20,838 singleton births without ART. The authors reported birth defects in 1,003 conceptions without ART $(4.8 \%)$ and in 235 infants conceived by ICSI (6.5\%) OR 1.41 (95\% CI [1.20-1.65]).

Davies et al. (2012) conducted a retrospective cohort study between January 1986 and December 2002. The authors they analyzed data from two infertility clinics in South Australia, both operated by the University of Adelaide and Flinders. Of 308,974 births, 1,878 resulted from conception by ICSI. The Odds ratio for any birth defects in ICSI groups (182 defects, 9.7\%) compared to pregnancies without ART in the general population $(16,989$ defects $5.7 \%$ ) was 2.27 (95\% CI [1.86-2.77]) for singleton pregnancies.
In the publication by Källén et al. (2005), data from infants born after ART in the 1982-2001 period of all assisted reproduction clinics in Sweden were retrospectively collected. Children from each subtype of ART (ICSI or IVF) were compared to births in the general population during the same time period. The total number of children born from singleton ICSI pregnancies was 4,949, with malformations occurring in $307(6.2 \%)$ infants; in the control population covering a total of $2,039,943$ infants, $80,881(4.0 \%)$ were born with malformations of any type OR 1.63 (95\% [CI 1.42-1.87]).

Belva et al. (2007) carried out a cross-sectional assessment of the medical outcomes of 8-year-old children born from singleton ICSI pregnancies $(n=150)$ after 32 weeks of gestation versus 147 children of the same age born from singleton pregnancies after spontaneous conception. Information on the general health status was obtained through a questionnaire sent to parents and physical examination performed by the authors. Fifteen of the $150(10 \%)$ ICSI children and five of the 147 (3.3\%) naturally conceived children had major congenital malformations. OR=2.84; (95\% CI [1.15-7.05]). Minor congenital malformations were also described in the ICSI group in $35 / 145$ (24.1\%) subjects versus $25 / 145$ (17.2\%) children in the control group.

Fedder et al. (2013) published a cross-sectional cohort study with 8-year-olds enrolling all children born after ICSI with testicular or epididymal semen and fresh embryo transfers in Denmark, from 1995 to 2009. The control group included children conceived by ICSI with ejaculated sperm and by natural conception. The case group had 488 children, while the control group included 63,854 naturally conceived children. Singleton pregnancies after ICSI with any subtype of sperm retrieval were analyzed in this review as part of the case group. Malformations were seen in $7.9 \%(488 / 6,157)$ of the individuals included in the ICSI group and in $3.0 \%(1,952 / 63,854)$ of the naturally conceived children, yielding an OR of 4.25 (95\% CI [3.684.90]).

Ludwig et al. (2009) performed a cross-sectional cohort study with children conceived by ICSI from singleton pregnancies aged 4-6 years. Information was collected from questionnaires filled out by the parents and examination of the infants performed from September 2004 to August 2006, in the German cities of Lübeck, Kiel, Hamburg, Magdeburg, Berlin, Bremen, Hannover, Essen, and Münster. Thirty-two reports of minor or major congenital malformation were accounted from the 275 children born after ICSI, but no statistically significant difference was found in relation to the control group, in which 24 cases of malformation were reported from a total of 272 children born after spontaneous conception $(11.6 \%$ vs. $8.8 \%)$, OR 1.35 (95\% CI [0.78-2.35]). No differences were seen in the specific incidence of major congenital malformations (7.6\% vs. $6.3 \%)$.

Sutcliffe et al. (2003) examined two cohorts of children born after ICSI, 56 infants aged 13-15 months in Australia and 208 in the United Kingdom. The control group was made up of 39 naturally conceived children from Australia and 221 from the United Kingdom. The data combined revealed similar levels of congenital malformation in the ICSI and naturally conceived individuals (5.6\% vs. $5.7 \%)$, $15 / 264$ vs. 15/260, OR 0.98 (95\% CI [0.47-2.05]) for singleton pregnancies.

In a cross-sectional study, Sutcliffe et al. (2001) analyzed a case group consisting of 208 children aged 1-2 years born after ICSI, based on data collected from 22 fertility clinics in the United Kingdom, and a control group with 221 naturally conceived children from January 1997 to January 1999. Ten major malformations were seen in the case group (4.8\%) and ten in the control group 
$(4.5 \%)$; and twenty-two (10.6\%) minor malformations were observed in the case group and $14(6.3 \%)$ in the control group. These values yielded a non-significant risk increase with an Odds Ratio for any congenital anomaly of 1.48 (95\% CI [0.84-2.60]).

Ombelet et al. (2005) performed a retrospective cohort study using data from the "Studiecentrum voor Perinatale Epidemiologie" (SPE). The SPE collects data from ongoing pregnancies, births, and the neonatal period in Flanders, from 22 weeks of gestation or birth weight $\geq 500 \mathrm{~g}$ ). A total of 430,565 births were recorded between January 1997 and December 2003, with 1,655 singleton births after ICSI. The analysis did not differentiate between major and minor malformations, and 3,278 naturally conceived pregnancies were studied as a control group. The congenital malformation rate was similar $(2.1 \%$ and $2.2 \%)$ in both groups, with 36 malformations in the ICSI group and 70 in the control group OR 1.01 (95\% CI [0.68-1.53]).

Place \& Englert (2003) published a longitudinal prospective study comparing the physical, psychomotor, and intellectual development of infants conceived by ICSI at the age of five years and spontaneously conceived infants. All children were born from singleton full-term pregnancies and attended at the Erasme Hospital Fertility Clinic in Brussels, Belgium. There was no statistically significant difference between both groups for the incidence of combined congenital malformations (10.6\% vs. $13.6 \%)$. (OR 0.75 (95\% CI [0.26-2.22]).

Hansen et al. (2012) conducted a retrospective cohort study from October 1996 to September 1999. Data were obtained from three databases to identify all children born after assisted reproductive technology $(2,911)$ procedures and births without the aid of assisted reproduction $(210,997)$. The study included all births with and without defects, pregnancy terminations due to fetal anomalies, and physical examination of infants at the age of six years. Major birth defects were diagnosed in $9.0 \%$ (53/586) of the infants born to singleton ICSI pregnancies and in 5.4\% of the infants born to singleton pregnancies without the aid of reproductive technologies $(11,078 / 205,641)$. OR 2.04 (95\% CI [1.43-2.93]).

A retrospective study by Klemetti et al. (2005) on children born to women offered ART (FIV, ICSI, and frozen embryo transfer) between October 1996 and September 1999 in Finland included 861 singleton pregnancies after ICSI from a total of 4,559 children born after IVF/ ICSI. The control group had 26,489 naturally conceived children born after singleton pregnancies. Major congenital malformations were found in $40(4.6 \%)$ children conceived by ICSI and in $756(2.8 \%)$ individuals in the control group, yielding an OR of 1.88 (95\% CI [1.25-2.82]).

Farhi et al. (2013) published a retrospective cohort study that included 2,646 singleton live births from ICSI and 202,935 births after spontaneous conception in the 1997-2004 time period. The study evaluated neonatal outcomes including congenital malformations. The authors reported increased risk for all congenital malformations in ICSI versus spontaneously conceived children $(2.5 \%$ vs. $1.8 \%)$, yielding an OR of 1.48 ; (95\% CI: [1.11-1.97]).

Funke et al. (2010) published a retrospective study that included infants born from January 1999 to December 2008 in the Department of Obstetrics and Gynecology of the Medical School of the University of Pécs, Hungary. A total of 15,206 births were recorded, with gestational ages ranging from 24 to 41 weeks, including 558 infants born from singleton pregnancies after ICSI. All children were examined at birth by neonatologists. Cases of urogenital malformation in males were detected and used for statistical analysis. Thirteen $(2.3 \%)$ males in the ICSI group and $155(1.1 \%)$ of the spontaneously conceived children were diagnosed with hypospadias or cryptorchidism. OR 3.05 (95\% CI [1.37-6.80]).

Bonduelle et al. (2004) performed a cross-sectional observational study as part of a controlled multicenter study carried out in three countries and compared children aged 5 years ( \pm 9 months) born of singleton pregnancies after ICSI to children born of singleton pregnancies after spontaneous conception. In the ICSI group, Nineteen children $(6.3 \%)$ in the ICSI group and eight $(3.0 \%)$ in the control group were diagnosed with major malformations, yielding a non-significant difference, OR 2.08; (95\% CI [0.99-4.50]).

Katalinic etal. (2004) carried out a controlled prospective multicenter study on a national scale in Germany between 1993 and 2001. The authors assessed fetuses and children born from singleton pregnancies, 2,055 from ICSI and 7,861 naturally conceived fetuses and children from 16 weeks of gestation in singleton pregnancies. The rate of major malformations was $8.9 \%(183 / 2,055)$ for the ICSI group, and $6.0 \%(472 / 7.861)$ for the control group, OR, 1.60 (95\% CI [1.31-1.94]).

And finally, Knoester et al. (2008) published a prospective cohort study from a clinical follow-up study performed at a University Medical Center between March 2004 and May 2005. The study examined children aged 5-8 years born to singleton pregnancies after ICSI between June 1996 and December 1999 and compared them to a control group including naturally conceived children born in the same time period. ICSI children showed no difference in the prevalence of major malformations when compared to naturally conceived children (6.9\% vs. $5.9 \%$, OR 1.18 95\% CI [0.35-4.00]).

The combined odds ratio of all studies developed to assess the risk of congenital malformations in ICSI-born infants versus the risk in naturally conceived infants is 1.99 (95\% CI [1.87-2.11]) (Figure 2 and Table 1).

\section{DISCUSSION}

In this systematic review of papers published between 2000 and 2016, we tried to answer a question that has echoed in the literature since ICSI was first offered to couples unable to conceive through traditional IVF. The analysis of the data from the 21 studies combined yielded an OR of 1.99 (95\% CI [1.87-2.11]) for congenital malformations in ICSI-conceived children when compared to naturally conceived children, which translates into an increased risk of $7.1 \%$ of having a malformation for individuals born after ICSI versus $4.0 \%$ for naturally conceived children.

However, one must be cautious when evaluating this result, as comparing data from different studies often brings important methodological implications and difficulties. More specifically, the data provided by general hospitals are likely to underestimate the risk of birth defects, since in birth defect registries the affected patients were not actively sought and data beyond birth were not reported. It should also be noted that records of birth defects arising from different assisted reproductive technologies may underestimate total risk, since patients are rarely followed after birth and, in some cases, data are reported only until the end of pregnancy and information on pregnancies under 20 weeks and miscarriages are not included. Another weakness of this review is the analysis of general data of the populations included in the study, since only papers reporting data for singleton pregnancies and with a naturally conceived control group were included, leaving aside many confounding factors not described in many of the publications, such as adjustment for maternal or paternal age, cause of male infertility, use of ejaculated or surgically obtained sperm, socio-economic status, use 


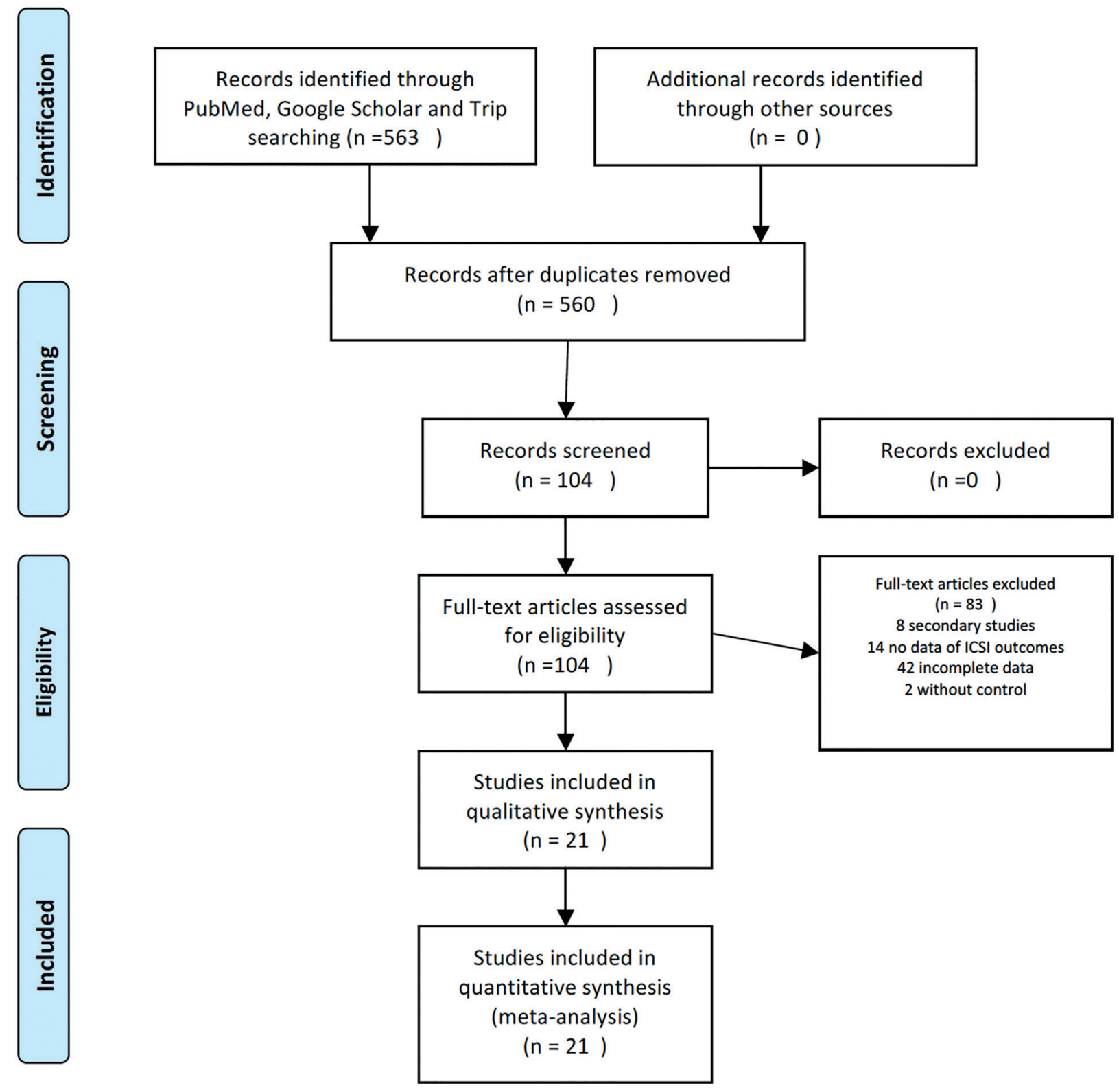

Figure 2. Forest plot analysis.

and abuse of substances or medication during pregnancy, smoking, parity, fresh vs. frozen embryo transfer, and the drug scheme used in ovarian hyperstimulation.

It has recently been suggested that an appropriate comparison group for children born after ICSI should include children born to infertile couples who conceived spontaneously without the aid of infertility treatment (Retzloff \& Hornstein, 2003). In practice this is not an easy group to find.

The strengths of this review were the adjustment of data for singleton pregnancies, avoiding the confusion of dizygotic or monozygotic multiple pregnancies; the large number of patients included in both groups, which gave this publication sufficient weight for it to be considered in the discussions in the literature on the actual risk of malformation introduced by ICSI; multinational and largescale studies were also included, with patients of different races and ethnicities, providing for a more general view that might be extrapolated to any population; and finally, the observed results were within the margins granted by existing publications in the medical literature.

This study included systematic reviews and metaanalyses that addressed aspects of our research question and allowed for comparisons between results. Some studies did not report increased risk of congenital malformations in the ICSI group, while others found twofold or greater increases in risk. The most relevant aspects of each study are described below.

The systematic review published by Hindryckx et al. (2010) was based on prospective follow-up studies with two-year-old children, and found a malformation rate after ICSI of $6.5 \%$. One weakness of this study was that the authors did not include a control group (IVF or naturally conceived). However, the rate of malformation reported in this study was within the range described in the literature and was also greater than the accepted baseline risk of $2-3 \%$ in the general population. In addition, one of the nine malformations found was hypospadias, which was 


\begin{tabular}{|c|c|c|c|c|c|c|c|}
\hline Author, year & Country & $\begin{array}{c}\text { Number } \\
\text { children } \\
\text { malformation } \\
\text { (ICSI) }\end{array}$ & $\begin{array}{c}\text { Number } \\
\text { children } \\
\text { malformation } \\
\text { (Control) }\end{array}$ & $\begin{array}{c}\text { Type of } \\
\text { Malformation }\end{array}$ & OR & $95 \%$ CI & Weight \\
\hline Hansen et al., 2002 & Australia & $18(9.7 \%)$ & $164(4.2 \%)$ & Major & 3.629 & $1.778-7.406$ & 0.76 \\
\hline Farhangniya et al., 2013 & Iran & $17(6.5 \%)$ & $29(4.4 \%)$ & Major & 1.525 & $0.792-2.935$ & 0.91 \\
\hline Bonduelle et al., 2005 & $\begin{array}{l}5 \text { European } \\
\text { countries }\end{array}$ & $178(33 \%)$ & $117(22 \%)$ & Both & 1.757 & $1.345-2.297$ & 5.43 \\
\hline $\begin{array}{l}\text { Sanchez-Albisua et al., } \\
2007\end{array}$ & Germany & $16(47 \%)$ & $8(20.5 \%)$ & Both & 3.275 & $1.239-8.660$ & 0.41 \\
\hline Halliday et al., 2010 & Australia & $235(6.5 \%)$ & $1003(4.8 \%)$ & Major & 1.411 & $1.201-1.657$ & 15.06 \\
\hline Davies et al., 2012 & Australia & $182(9.7 \%)$ & $\begin{array}{l}16989 \\
(5.4 \%) \\
\end{array}$ & Major & 2.276 & $1.864-2.778$ & 9.78 \\
\hline Källén et al., 2005 & Sweden & $307(5.8 \%)$ & $\begin{array}{l}80881 \\
(3.4 \%)\end{array}$ & Major & 1.636 & $1.424-1.879$ & 20.2 \\
\hline Belva et al., 2007 & Belgium & $15(10 \%)$ & $5(3.4 \%)$ & Both & 2.849 & $1.151-7.052$ & 0.47 \\
\hline Fedder et al., 2013 & Denmark & $488(7.9 \%)$ & $1952(3.0 \%)$ & Major & 4.252 & $3.687-4.904$ & 19.14 \\
\hline Sutcliffe et al., 2003 & $\begin{array}{l}\text { Australia } \\
\text { and UK }\end{array}$ & $15(5.7 \%)$ & $15(5.8 \%)$ & Minor & 0.984 & $0.471-2.055$ & 0.72 \\
\hline Sutcliffe et al., 2001 & UK & $32(15.3 \%)$ & $24(10.8 \%)$ & Both & 1.488 & $0.849-2.609$ & 1.24 \\
\hline Ombelet et al., 2005 & Belgium & $36(2.2 \%)$ & $70(2.1 \%)$ & Major & 1.019 & $0.678-1.532$ & 2.34 \\
\hline Place \& Englert, 2003 & Belgium & $7(10.6 \%)$ & $8(13.5 \%)$ & Both & 0.758 & $0.258-2.223$ & 0.34 \\
\hline Hansen et al., 2012 & Australia & $53(9.0 \%)$ & $\begin{array}{l}11078 \\
(5.4 \%) \\
\end{array}$ & Major & 2.047 & $1.430-2.930$ & 3.02 \\
\hline Klemetti et al., 2005 & Finland & $40(4.6 \%)$ & $756(2.8 \%)$ & Major & 1.885 & $1.259-2.823$ & 2.39 \\
\hline Farhi et al., 2013 & Israel & $67(2.5 \%)$ & $3700(1.8$ & Major & 1.483 & $1.114-1.974$ & 4.76 \\
\hline Funke et al., 2010 & Hungary & $13(2.3 \%)$ & $155(1.1 \%)$ & Major & 3.055 & $1.372-6.800$ & 0.61 \\
\hline Bonduelle et al., 2004 & $\begin{array}{l}\text { Belgium, } \\
\text { Sweden } \\
\text { and USA }\end{array}$ & $19(6.3 \%)$ & $8(3.0 \%)$ & Major & 2.077 & $0.958-4.502$ & 0.65 \\
\hline Katalinic et al., 2004 & Germany & $183(8.9 \%)$ & $472(6.0 \%)$ & Major & 1.600 & $1.316-1.946$ & 10.18 \\
\hline Knoester et al., 2007 & $\begin{array}{c}\text { The } \\
\text { Netherlands }\end{array}$ & $6(6.9 \%)$ & $5(5.9 \%)$ & Both & 1.183 & $0.350-4.001$ & 0.26 \\
\hline Total & & & & & 1.987 & 1.867-2.115 & $100 \%$ \\
\hline
\end{tabular}

highly associated with ICSI. However the study population was too small to draw definitive conclusions.

In 2008, Bertelsmann et al. (2008) published a systematic review and meta-analysis in which the authors found that the variability of malformations rates reported among the included studies was considerably high. They concluded that the rates of reported malformations depend on both the used categorization system, the time at which the children were examined, and the method of examination performed (physical only or accompanied by auxiliary tests). In addition, the authors found that the rate of malformations might be strongly impacted, for example, by the selection of embryos prior to transfer and by induction abortion secondary to pathological prenatal findings. The rate of malformations after assisted fertilization is low in all studies they analyzed; but most studies reported rates of malformation only for live births, without taking into account the cases occurred in stillbirths and induction abortions, as recommended by the EUROCAT.

Hansen et al. (2005) published the first systematic review and meta-analysis that reviewed and put together epidemiological data to assess the risk of birth defects following the use of assisted reproductive technologies.
The literature search identified 25 documents with data on birth defects after IVF/ICSI compared to spontaneously conceived children. Their results suggest that there is a statistically significant higher risk of birth defects in ICSIconceived infants in the order of $30-40 \%$. All selected studies adjusted their data by maternal age and parity, but other factors such as sex of the baby, year of birth, and plurality were adjusted by the authors for publication in this review. However, information on other potential confounders such as maternal exposure to toxins and socioeconomic level were not available in most studies. It should be noted that the same authors wrote one of the included papers in 2002.

Rimm et al. (2004) performed a meta-analysis in which the parameters used for risk estimation included birth weight, prematurity, minor and major congenital malformations, developmental anomalies, chromosomal abnormalities, multiple births, and perinatal mortality. In terms of congenital malformations combined, the authors reported a global OR of 1.29; but they also noted significant design differences between the 19 studies included in their analysis, such as time of examination of the newborns, lack of adjustment for maternal age and other factors, 
methods used to assess infants in the case and control groups, and the type of control group, since all included studies used a general population control group, when the most appropriate option, according to the authors, would have been to include infertile couples who eventually conceived spontaneously without infertility treatment.

Lie et al. (2005) published a meta-analysis in which weak evidence of increased risk of birth defects was found after ICSI versus standard IVF. This indicates, in Lie's view, that the microinjection procedure used in ICSI does not represent a large increase in the risk of birth defects when compared to IVF in general. In this review, with a power of $80 \%$, a relative risk of 1.4 was detected for cardiovascular defects, 1.6 for musculoskeletal defects, 1.8 for hypospadias, 2.0 for cleft lip or cleft palate, and 2.2 for neural tube defects, with a significance level of $5 \%$. The inclusion criteria and definitions of major defects differed between the studies included in the analysis. Information on other potential confounders such as smoking, maternal age, among others, was not available in most studies, but the potential for confounding may also be small in these categories. The risk ratio for all data was estimated at 1.09 (CI: 0.86 to 1.36 ), so this meta-analysis found no significant increase in the risk of birth defects for ICSIconceived infants when compared to standard IVF.

Wen et al. (2012) published a report in which they grouped epidemiological data to assess the risk of birth defects after ART and compared the difference in risk of birth defects after ICSI or IVF to that of the general population. Their results suggest that there is a significant increase in the risk of birth defects in babies conceived by ART, but not in the ones conceived by ICSI when compared the individuals conceived after traditional IVF. They reported considerable heterogeneity among the 56 studies included in their analysis. Differences included methodology for assessing case and control infants, as well as lack of adjustment for maternal age and other factors. They argued that the excess risk of birth defects found in children born after ART might be due to the underlying infertility of couples seeking treatment, rather than the mechanisms used in ART. The main limitation of the study is that the in vitro fertilization and/or ICSI groups were compared to naturally conceived children, rather than babies born to infertile couples who conceived without ART, as also seen in our review.

Finally, Qin et al. (2015) found that children born after ICSI were at increased risk of congenital malformations in relation to individuals conceived naturally $(R R=1.33)$. Risk increased even more when the data were restricted to single births only $(R R=138)$, when major malformations were assessed $(R R=1.47)$, when adjusted studies were performed $(R R=1.37)$, and when only high quality studies were analyzed $(R R=1.40)$. Subgroup analysis showed an increased risk of malformations in single births. When compared to spontaneous conception, available data supported that ICSI pregnancies had increased risk for adverse outcomes such as prenatal hemorrhage, pregnancy hypertension, premature rupture of membranes, cesarean section, perinatal mortality, gestational diabetes, labor induction, preterm birth, extreme preterm birth, low birth weight, very low birth weight, and small for gestational age. However, the association between ICSI and increased risk of congenital malformations was controversial. It is noteworthy that the included studies were difficult to interpret due to methodological problems, including inadequate case sizes, lack of adequate data to compare, and inconsistent methods used for the detection of congenital malformations in the case and control groups. All original studies included in this review used a prospective cohort design, which minimized selection and recall biases. There were several limitations. Some studies included infants born from ovulation induction in the control group of spontaneous conception, while other studies did not differentiate between major and minor malformations.

Similarly to our study, these results (despite the differences in adjustment factors and control populations) also pointed out to, in our opinion, a biologically plausible increased risk of birth defects in ICSI-conceived babies. Factors associated with reproductive management that may increase the risk of birth defects include the underlying causes of infertility in couples seeking treatment and factors associated with IVF/ICSI procedures per se, such as freezing and thawing embryos, late fertilization of oocytes, the composition of the culture medium, and the drugs used to induce ovulation or to support the luteal phase (Hansen et al., 2005)

Finally, combining major and minor defects may lead to less precise risk estimation, since minor defects are often incompletely reported and/or underreported. Nevertheless, only $67 \%$ of the studies in our review provided separate data on major birth defects. Since there appears to be a greater risk of birth defects in babies born after conception by ICSI and that the cause cannot yet be fully identified, it is very important that future studies consider the details pertaining to the treatments offered to couples and the underlying causes of infertility, along with the adjustment of all biographical data that might potentially affect the outcomes.

The results of our systematic review and meta-analysis suggest that infants born from singleton pregnancies after ICSI are at increased risk of developing congenital malformations when compared to similar naturally conceived children. These results may reflect the existence of real risk stemming from the mode of ART due to gamete manipulation, embryo freezing and thawing, and the composition of the culture media, in addition to factors related to parent characteristics such as maternal or paternal age, male factor infertility, and other variables not analyzed in the present study such as socio-economic status, use of substances during pregnancy, the method used to induce ovarian hyperstimulation, and others. In order to offer sound advice to couples looking at ICSI, our findings should be considered along with those of others. In a recently published study, Belva et al. (2016) found after correcting for several confounding factors - almost twice the sperm concentration in spontaneously conceived peers in comparison to ICSI men.

\section{CONFLICT OF INTEREST}

The authors have no conflicts of interest to declare.

\section{Corresponding author:}

Juan Enrique Schwarze

Reproductive Medicine Unit at Clinica Monteblanco

Santiago - Chile

E-mail: jeschwarze@gmail.com

\section{REFERENCES}

Belva $F$, Henriet $S$, Liebaers I, Van Steirteghem A, Celestin-Westreich S, Bonduelle M. Medical outcome of 8-year-old singleton ICSI children (born >or $=32$ weeks' gestation) and a spontaneously conceived comparison group. Hum Reprod. 2007;22:506-15. PMID: 16982659 DOI: $10.1093 /$ humrep/del372

Belva $\mathrm{F}$, Bonduelle $\mathrm{M}$, Roelants $\mathrm{M}$, Michielsen $\mathrm{D}$, Van Steirteghem A, Verheyen G, Tournaye H. Semen quality of young adult ICSI offspring: the first results. Hum Reprod. 2016;31:2811-20. PMID: 27707840 DOI: $10.1093 /$ humrep/dew245 
Bertelsmann H, de Carvalho Gomes H, Mund M, Bauer $\mathrm{S}$, Matthias $\mathrm{K}$. The risk of malformation following assisted reproduction. Dtsch Arztebl Int. 2008;105:11-7. PMID: 19578448 DOI: 10.3238/arztebl.2008.0011

Bonduelle M, Bergh C, Niklasson A, Palermo GD, Wennerholm UB; Collaborative Study Group of Brussels, Gothenburg and New York. Medical follow-up study of 5-year-old ICSI children. Reprod Biomed Online. 2004;9:91-101. PMID: 15257828

Bonduelle M, Wennerholm UB, Loft A, Tarlatzis BC, Peters C, Henriet S, Mau C, Victorin-Cederquist A, Van Steirteghem A, Balaska A, Emberson JR, Sutcliffe AG. A multi-centre cohort study of the physical health of 5-yearold children conceived after intracytoplasmic sperm injection, in vitro fertilization and natural conception. Hum Reprod. 2005;20:413-9. PMID: 15576393 DOI: $10.1093 /$ humrep/deh592

Davies MJ, Moore VM, Willson KJ, Van Essen P, Priest $K$, Scott H, Haan EA, Chan A. Reproductive technologies and the risk of birth defects. N Engl J Med. 2012;366:1803-13. PMID: 22559061 DOI: $10.1056 /$ NEJMoa1008095

Ericson A, Källén B. Congenital malformations in infants born after IVF: a population-based study. Hum Reprod. 2001;16:504-9. PMID: 11228220 DOI: http://dx.doi.org/10.1093/humrep/16.3.504

Farhangniya M, Dortaj Rabori E, Mozafari Kermani R, Haghdoost AA, Bahrampour A, Bagheri P, A L Lancaster P, Ashrafi M, Vosough Taqi Dizaj A, Gourabi H, Shahzadeh Fazeli A. Comparison of Congenital Abnormalities of Infants Conceived by Assisted Reproductive Techniques versus Infants with Natural Conception in Tehran. Int J Fertil Steril. 2013;7:217-24. PMID: 24520489

Farhi A, Reichman B, Boyko V, Mashiach S, Hourvitz A, Margalioth EJ, Levran D, Calderon I, Orvieto R, Ellenbogen A, Meyerovitch J, Ron-El R, Lerner-Geva L. Congenital malformations in infants conceived following assisted reproductive technology in comparison with spontaneously conceived infants. J Matern Fetal Neonatal Med. 2013;26:1171-9. PMID: 23451839 DOI: $10.3109 / 14767058.2013 .776535$

Fedder J, Loft A, Parner ET, Rasmussen S, Pinborg A. Neonatal outcome and congenital malformations in children born after ICSI with testicular or epididymal sperm: a controlled national cohort study. Hum Reprod. 2013;28:230-40. PMID: 23154066 DOI: $10.1093 /$ humrep/des377

Funke S, Flach E, Kiss I, Sándor J, Vida G, Bódis J, Ertl T. Male reproductive tract abnormalities: more common after assisted reproduction? Early Hum Dev. 2010;86:547-50. PMID: 20674196 DOI: 10.1016/j.earlhumdev.2010.06.015

Halliday JL, Ukoumunne OC, Baker HW, Breheny S, Jaques AM, Garrett C, Healy D, Amor D. Increased risk of blastogenesis birth defects, arising in the first 4 weeks of pregnancy, after assisted reproductive technologies. Hum Reprod. 2010;25:5965. PMID: 19850591 DOI: 10.1093/humrep/dep364

Hansen M, Kurinczuk JJ, Bower C, Webb S. The risk of major birth defects after intracytoplasmic sperm injection and in vitro fertilization. N Engl J Med. 2002;346:725-30. PMID: 11882727 DOI: $10.1056 /$ NEJMoa010035
Hansen M, Bower C, Milne E, de Klerk N, Kurinczuk JJ. Assisted reproductive technologies and the risk of birth defects-a systematic review. Hum Reprod. 2005;20:32838. PMID: 15567881 DOI: 10.1093/humrep/deh593

Hansen M, Kurinczuk JJ, de Klerk N, Burton P, Bower C. Assisted reproductive technology and major birth defects in Western Australia. Obstet Gynecol. 2012;120:852-63. PMID: 22996103 DOI: 10.1097/AOG.0b013e318269c282

Hindryckx A, Peeraer $K$, Debrock S, Legius $E$, de Zegher $F$, Francois I, Vanderschueren D, Demyttenaere K, Rijkers A, D'Hooghe T. Has the prevalence of congenital abnormalities after intracytoplasmic sperm injection increased? The Leuven data 1994-2000 and a review of the literature. Gynecol Obstet Invest. 2010;70:11-22. PMID: 20110727 DOI: $10.1159 / 000279323$

Jwa J, Jwa SC, Kuwahara A, Yoshida A, Saito H. Risk of major congenital anomalies after assisted hatching: analysis of three-year data from the national assisted reproduction registry in Japan. Fertil Steril. 2015;104:71-8. PMID: 25935490 DOI: $10.1016 /$ j.fertnstert.2015.03.029

Källén $B$, Finnström $O$, Nygren KG, Olausson PO. In vitro fertilization (IVF) in Sweden: risk for congenital malformations after different IVF methods. Birth Defects Res A Clin Mol Teratol. 2005;73:162-9. PMID: 15678490 DOI: $10.1002 /$ bdra. 20107

Katalinic A, Rösch C, Ludwig M; German ICSI FollowUp Study Group. Pregnancy course and outcome after intracytoplasmic sperm injection: a controlled, prospective cohort study. Fertil Steril. 2004;81:1604-16. PMID: 15193484 DOI: $10.1016 /$ j.fertnstert.2003.10.053

Klemetti R, Gissler M, Sevón T, Koivurova S, Ritvanen A, Hemminki E. Children born after assisted fertilization have an increased rate of major congenital anomalies. Fertil Steril. 2005;84:1300-7. PMID: 16275218 DOI: $10.1016 /$ j.fertnstert.2005.03.085

Knoester M, Helmerhorst FM, Vandenbroucke JP, van der Westerlaken LA, Walther FJ, Veen S; Leiden Artificial Reproductive Techniques Follow-up Project (L-art-FUP). Perinatal outcome, health, growth, and medical care utilization of 5- to 8-year-old intracytoplasmic sperm injection singletons. Fertil Steril. 2008;89:1133-46. PMID: 18177652 DOI: $10.1016 /$ j.fertnstert.2007.04.049

Lie RT, Lyngstadaas A, Ørstavik KH, Bakketeig LS, Jacobsen G, Tanbo T. Birth defects in children conceived by ICSI compared with children conceived by other IVF-methods; a meta-analysis. Int J Epidemiol. 2005;34:696-701. PMID: 15561745 DOI: $10.1093 /$ ije/dyh363

Ludwig AK, Katalinic A, Thyen U, Sutcliffe AG, Diedrich $K$, Ludwig M. Physical health at 5.5 years of age of termborn singletons after intracytoplasmic sperm injection: results of a prospective, controlled, single-blinded study. Fertil Steril. 2009;91:115-24. PMID: 18206144 DOI: $10.1016 /$ j.fertnstert.2007.11.037

Massaro PA, MacLellan DL, Anderson PA, Romao RL. Does intracytoplasmic sperm injection pose an increased risk of genitourinary congenital malformations in offspring compared to in vitro fertilization? A systematic review and meta-analysis. J Urol. 2015;193:1837-42. PMID: 25813561 DOI: $10.1016 /$ j.juro.2014.10.113 
Ombelet W, Peeraer K, De Sutter P, Gerris J, Bosmans E, Martens G, Ruyssinck G, Defoort P, Molenberghs G, Gyselaers W. Perinatal outcome of ICSI pregnancies compared with a matched group of natural conception pregnancies in Flanders (Belgium): a cohort study. Reprod Biomed Online. 2005;11:244-53. PMID: 16168226 DOI: $10.1016 / \mathrm{S} 1472-6483(10) 60965-0$

Place I, Englert Y. A prospective longitudinal study of the physical, psychomotor, and intellectual development of singleton children up to 5 years who were conceived by intracytoplasmic sperm injection compared with children conceived spontaneously and by in vitro fertilization. Fertil Steril. 2003;80:1388-97. PMID: 14667874 DOI: 10.1016/j.fertnstert.2003.06.004

Qin J, Sheng X, Wang H, Liang D, Tan H, Xia J. Assisted reproductive technology and risk of congenital malformations: a meta-analysis based on cohort studies. Arch Gynecol Obstet. 2015;292:777-98. PMID: 25877221 DOI: $10.1007 / \mathrm{s} 00404-015-3707-0$

Retzloff MG, Hornstein MD. Is intracytoplasmic sperm injection safe? Fertil Steril. 2003;80:851-9. PMID: 14556800 DOI: $10.1016 /$ S0015-0282(03)01014-8

Rimm AA, Katayama AC, Diaz M, Katayama KP. A meta-analysis of controlled studies comparing major malformation rates in IVF and ICSI infants with naturally conceived children. J Assist Reprod Genet. 2004;21:43743. PMID: 15704519 DOI: $10.1007 /$ s10815-004-8760-8

Sanchez-Albisua I, Borell-Kost S, Mau-Holzmann UA, Licht $P$, Krägeloh-Mann I. Increased frequency of severe major anomalies in children conceived by intracytoplasmic sperm injection. Dev Med Child Neurol. 2007;49:129-34. PMID: 17254001 DOI: 10.1111/j.1469-8749.2007.00129.x
Sutcliffe AG, Taylor B, Saunders K, Thornton S, Lieberman BA, Grudzinskas JG. Outcome in the second year of life after in-vitro fertilisation by intracytoplasmic sperm injection: a UK case-control study. Lancet. 2001;357:2080-4. PMID: 11445100 DOI: $10.1016 /$ S0140-6736(00)05180-1

Sutcliffe AG, Saunders K, McLachlan R, Taylor B, Edwards P, Grudzinskas G, Leiberman B, Thornton S. A retrospective case-control study of developmental and other outcomes in a cohort of Australian children conceived by intracytoplasmic sperm injection compared with a similar group in the United Kingdom. Fertil Steril. 2003;79:512-6. PMID: 12620432 DOI: 10.1016/S0015-0282(02)04701-5

Tararbit K, Lelong N, Thieulin AC, Houyel L, Bonnet D, Goffinet F, Khoshnood B; EPICARD Study Group. The risk for four specific congenital heart defects associated with assisted reproductive techniques: a populationbased evaluation. Hum Reprod. 2013;28:367-74. PMID: 23178272 DOI: 10.1093/humrep/des400

Wen J, Jiang J, Ding C, Dai J, Liu Y, Xia Y, Liu J, Hu Z. Birth defects in children conceived by in vitro fertilization and intracytoplasmic sperm injection: a meta-analysis. Fertil Steril. 2012;97:1331-7.e1-4. PMID: 22480819 DOI: $10.1016 /$ j.fertnstert.2012.02.053

Wennerholm UB, Bergh C, Hamberger L, Lundin K, Nilsson L, Wikland $M$, Källén B. Incidence of congenital malformations in children born after ICSI. Hum Reprod. 2000;15:944-8. PMID: 10739847 DOI: 10.1093/humrep/15.4.944 